\title{
Tarifsozialpolitik und Insider-Solidarität
}

In jüngster Zeit neigen politische Akteure verstärkt dazu, den Tarifparteien eine größere Verantwortung für die soziale Sicherung zuzuweisen. Augenfällig wird das etwa in der aktuellen Debatte über die zukünftige Form der Alterssicherung, bei der u. a. die tarifliche/ betriebliche Altersvorsorge die Lücke schließen soll, die sich durch die Absenkung des Niveaus der gesetzlichen Rente öffnen wird. Auch aus der Gestaltung des Erwerbsausstiegs zieht sich der Gesetzgeber zurück und überlässt das Feld den Tarifparteien. Der Beitrag thematisiert die im politischen Diskurs weitgehend vernachlässigte Frage, ob die Sozialpartner zu dieser Kompensation in der Lage sind und welche Konsequenzen die „Vertariflichung“" sozialer Sicherung für die Beschäftigten hat.

THILO FEHMEL

\section{Einleitung}

Seit einigen Jahren vollzieht sich ein Umbau des Sozialstaates, der sich in mehreren Dimensionen beobachten lässt. Für das hier zu behandelnde Thema der Tarifsozialpolitik sind zwei Aspekte besonders relevant: Die „Vermarktlichung“ sozialer Sicherung beschreibt den Übergang vom Wohlfahrtsstaat zum Wohlfahrtsmarkt (Nullmeier 2004; Leisering/Marschallek 2010). Soziale Sicherheit soll verstärkt durch marktförmige Wohlfahrtsproduktion anstelle staatlicher Wohlfahrtsgarantien realisiert werden. Zunehmend ist die Aufgabe staatlicher Sozialpolitik nicht mehr unmittelbare öffentliche Umverteilung, sondern - unter (politisch verstärkt disputablen) sozialpolitischen Gesichtspunkten die Schaffung und Regulierung von Märkten, auf denen Güter und Dienstleistungen sozialer Wohlfahrt angeboten und nachgefragt werden (Nullmeier 2001). Wem die individuelle Risikoabsicherung am Wohlfahrtsmarkt nicht gelingt, dem werden zunehmend nurmehr existenzsichernde Grundsicherungsleistungen anstatt lebensstandardsichernder Sozialleistungen in Aussicht gestellt. Der damit einhergehende Wandel der Einnahmen- und Ausgabenanteile des traditionell beitragsdominierten, lohnarbeitszentrierten Sozialstaates Bismarckscher Prägung wird beschrieben als Dualisierung sozialer Sicherung - einerseits beitragsfinanzierte und beitragsäquivalente Leistungen für ArbeitsmarktInsider, andererseits ein wachsender Anteil steuerfinanzier- ter Unterstützungsformen auf Mindestniveau für prekär Beschäftigte und Arbeitsmarkt-Outsider (Palier/Martin 2007).

Beide Entwicklungen werden, wie ich zeigen werde, von einem weiteren Trend sozialstaatlichen Umbaus begünstigt: der „Vertariflichung“ sozialer Sicherung. Darunter ist die zunehmende Überantwortung der Wohlfahrtsproduktion an die kollektiven Akteure des Systems der industriellen Beziehungen zu verstehen, also an eine Aushandlungs- und Gestaltungsebene, die sich durch ihre Eigengesetzlichkeiten von Sozialstaatlichkeit ebenso deutlich unterscheidet wie vom Handeln individueller Akteure auf Wohlfahrtsmärkten. Die Beteiligung der Tarifpartner an der Wohlfahrtsproduktion ist für sich genommen nichts Neues. Neu ist, dass die von Tarif- und Betriebsakteuren ausgehandelten Elemente sozialer Sicherung vermehrt substitutiv statt komplementär $\mathrm{zu}$ sozialstaatlichen Leistungen fungieren sollen (Bispinck 2012). Im vorliegenden Beitrag wird es nicht um die - triviale, aber keineswegs belanglose - Tatsache gehen, dass in den Genuss tariflicher und betrieblicher Sozialpolitik nur kommt, wer überhaupt und hinreichend lange als Arbeitnehmer im Geltungsbereich entsprechender Regelungen beschäftigt ist; dass also diese Form nichtstaatlicher Sozialpolitik auf Arbeitsmarkt-Insider beschränkt bleibt und insofern hochgradig selektiv ist. Mir geht es vielmehr um die Folgen der „Vertariflichung“ sozialer Sicherheit für die potenziell begünstigten Arbeitsmarkt-Insider selbst, um deren Handlungsoptionen vor dem Hintergrund strukturel- 
len Wandels und um die verteilungspolitischen Konsequenzen, die sich daraus langfristig ergeben.

Ich gehe wie folgt vor: Zunächst beleuchte ich das Verhältnis von Tarifsystem und staatlicher Sozialpolitik; dabei werden historische Prozesse der funktionalen Differenzierung beider Systeme ebenso nachgezeichnet wie deren in jüngerer Zeit zu beobachtende partielle Entdifferenzierung (Abschnitt 2). Dann diskutiere ich die Folgen dieser Entdifferenzierungsprozesse (3). In diesem Zusammenhang frage ich zunächst, was die „Vertariflichung“ sozialer Sicherheit für die Gewerkschaften selbst bedeutet (3.1) und stelle anschließend Überlegungen an zu den daraus resultierenden Konsequenzen für die Beschäftigten (3.2) wie für deren Solidaritätsbereitschaften (3.3). Abschließend wird die „Vertariflichung" sozialer Sicherung mit den anderen, oben genannten Entwicklungen in Beziehung gesetzt (4).

\section{Das dynamische Verhältnis von Sozialpolitik und Tarifsystem}

Das Wissen um die Interdependenz der Systeme der industriellen Beziehungen und der staatlichen sozialen Sicherung ist so alt wie die Sozialstaatsforschung selbst. Dass die sozialen Folgen der Industrialisierung wesentliche Triebkräfte für die Anfänge von Sozialstaatlichkeit waren, ist über alle theoretischen Schulen und Ansätze hinweg unstrittig. Sollten in Deutschland sozialpolitische Maßnahmen des nicht-demokratischen Staates zunächst häufig die kollektive Macht der Industriearbeiter abwehren und ihr die systemgefährdende Spitze nehmen, so schlugen sich schon in der Zwischenkriegszeit (Briefs 1930, S. 149), vor allem aber in der Expansionsphase des demokratischen Wohlfahrtsstaates nach dem Zweiten Weltkrieg bis in die 1980er Jahre hinein gewerkschaftliche Erfolge im Bereich qualitativer Tarifpolitik oft in entsprechender sozialstaatlicher Regulierung nieder (Wiesenthal/Clasen 2003, S. 297ff.). Umgekehrt strukturieren diese sozialstaatlichen Regularien ihrerseits wiederum die Handlungsbedingungen der Sozialpartner. Gewerkschaftliche Verhandlungs- und Kampffähigkeit basiert wesentlich auf zuvor erreichten tariflichen Standards, die durch ihre Überführung in Sozial- oder Arbeitsrecht als vergleichsweise dauerhaft institutionalisiert gelten können und nicht immer wieder aufs Neue in industriellen Konflikten erkämpft bzw. verteidigt werden müssen. Auf diese Weise hält Sozialpolitik zwei Konfliktarenen auseinander und reduziert - auch im Interesse der Arbeitgeber an berechenbaren Beziehungen - das Ausmaß von Verteilungskonflikten. Aus Klassenkonflikten werden politische, und das heißt: politisch handhabbare Konflikte (Lenhardt/Offe 1977, S. 110f.). Diese sozialstaatlich ermöglichte Befreiung der Gewerkschaften von permanenten Kämpfen um den Status quo setzt Ressourcen frei, die für die Durchsetzung weitergehender lohn- und sozialpolitischer Forderungen verwendet werden können. Insofern ist staatliche Sozialpolitik wesentliche Bedingung für ein modernes Tarifsystem.

Keineswegs jedoch ist der Sozialstaat nur die subalterne Exekutive der Aushandlungsergebnisse der industriellen Sozial- und Konfliktpartnerschaften. Staatliche Akteure ziehen, erstens, selbst durchaus Nutzen aus einer funktionsfähigen und legitimierten Tarifautonomie: „Mit dem frei ausgehandelten Tarifvertrag entlastet sich der Staat nicht nur von Ansprüchen und Erwartungen, die sich auf ihn richten, er erhöht seine eigenen Chancen der Legitimitätsgewinnung. Für eine politische Ordnung kommt es darauf an, dass Verteilungskonflikte nicht sofort zu Verfassungskonflikten werden." (Lepsius 1995, S. 6f.) Gerade weil - und wenn - die Tarifautonomie auf Anpassungsbedarfe im Wirtschaftssystem elastischer reagieren kann als staatliche Gesetzgebung, sind staatliche Akteure am Bestand eines solchen elastischen Tarifsystems mit gesamtgesellschaftlicher Strukturierungskraft interessiert und für seine grundsätzliche Bestandserhaltung auch unter Bedingungen ausgebauter Sozialstaatlichkeit zu vielem bereit. Insofern ist $\mathrm{Ta}$ rifautonomie wesentliche Stabilitätsbedingung staatlicher Politik. Vom Sozialstaat als bloßem Ausführungsorgan kann also nicht die Rede sein. Eher ist das Verhältnis von Tarifsystem und Sozialstaat als ein wechselseitig entlastendes und zudem arbeitsteiliges zu begreifen: Den Verbänden obliegt die Einkommensgestaltung der Beschäftigten innerhalb, dem Sozialstaat die Einkommenssicherung außerhalb des Arbeitsmarktes. Für beide Seiten sind die jeweiligen Erfolge dabei wesentliches Mittel der Machtreproduktion.

Aber: Dieses Verhältnis ist asymmetrisch zugunsten des Sozialstaates. Denn staatliche Akteure verfügen, zweitens, über vielfältige Möglichkeiten, das Verhältnis von Tarifsystem und staatlicher Sozialpolitik zu modifizieren (Fehmel 2010). Darüber, was sozialpolitisch angemessen ist, entscheiden sie situativ, und zwar entsprechend ihrer Einschätzung der eigenen Betroffenheit, die sich aus den politischen und fiskalischen Folgen ihrer sozialpolitischen Interventionen in wirtschaftliche Abläufe ergibt. Auf diese Weise strukturiert der Staat zugleich die Arbeitsbedingungen selbst, zum Beispiel durch die Definition von faktischen Lohnuntergrenzen mittels Arbeitslosenunterstützung oder Mindestlohn, durch die Festlegung von Renteneintrittsalter und Rentenhöhe, durch die Öffnung oder Schließung von Bildungssystemen als arbeitsmarktexternen Lebensbereichen u. ä. Gemeinsam ist diesen Beispielen, dass mit ihnen das Verhältnis von Angebot und Nachfrage am Arbeitsmarkt quantitativ reguliert werden kann, und zwar - je nach Arbeitskräftebedarf am Arbeitsmarkt - in beide Richtungen, also sowohl de- als auch re-kommodifizierend (Lenhardt/ Offe 1977, S. 110). Grundsätzlich haben staatliche Akteure also die Macht, den Ausbaugrad der Sozialpolitik zu bestimmen; und wo die Gestaltung des Verhältnisses von Deund Re-Kommodifizierung prinzipiell auch mit tarifpolitischen Mitteln möglich ist, strukturiert diese staatliche Macht damit zugleich die Handlungsbedingungen der Sozialpartner sowie deren Abhängigkeitsverhältnis vom Staat 
(Behrens 2005). Inwieweit staatliche Akteure dies in Abstimmung mit den Verbänden tun können oder gegen deren Widerstand durchsetzen müssen, ist eine empirisch stets erneut zu klärende Frage.

In jedem Fall aber verschiebt sich im Zuge solcher Entwicklungen im modernen, von kollektiven Akteuren geprägten Wohlfahrtsstaat die Grenze zwischen Sozialstaat und Tarifsystem, zwischen sozialstaatlich verantworteter und tarifpolitisch herzustellender sozialer Sicherheit. Wenn und soweit Sozialpolitik gesellschaftliche Prozesse funktionaler Differenzierung flankiert, indem sie das ökonomische System von Sicherheits- und Gerechtigkeitsansprüchen abschirmt und so dessen Konzentration auf Rentabilität und ökonomische Effizienzsteigerungen ermöglicht (Hockerts 1998, S. 20f.), dann ist die hier beschriebene „Vertariflichung“ (und „Verbetrieblichung“) der Gestaltung sozialer Sicherung in letzter Konsequenz als funktionale Entdifferenzierung zu verstehen. Zumindest aber steigt durch derartige Entwicklungen die Wahrscheinlichkeit von intersystemischen Kollisionen (Vobruba 1991, S. 123), da die von materieller Existenzsicherung abhängigen Erwerbstätigen ihre Ansprüche wieder verstärkt unmittelbar an die Unternehmen, also an das ökonomische System richten müssen. Das kommt einer Re-Integration der beiden Konflikt- und Verteilungsarenen Arbeitsmarkt und Sozialpolitik gleich. Ursache dieser Prozesse ist der Wandel des staatlichen Selbstverständnisses: weg von einem eigenständigen oder kooperativ koordinierenden aktiven Gestaltungshandeln hin zu einem Akteur, der sich darauf beschränkt, das Gestaltungshandeln anderer zu regulieren bzw. die Selbstregulierung anderer zu beaufsichtigen (Vogel 2007, S. 47ff.). Diese Form staatlichen Rückzugs aus Gestaltungsfragen sozialer Sicherung geht über die von Hall (1993) systematisierten Stufen sozialpolitischen Wandels erster und zweiter Ordnung weit hinaus: Der Rückzug bedeutet die Aufgabe der weitgehend alleinigen sozialpolitischen Verantwortung des Staates. Der Paradigmenwechsel zeigt sich in Formen der Dekommodifizierung, die vormals (primär) sozialstaatlich organisiert wurden, nun aber im Zuge der Entstaatlichung sozialer Sicherung zunehmend zur Domäne tarifpolitischer Regulierung werden müssen.

Am deutlichsten sichtbar wird diese Verschiebung in jenem sozialpolitischen Bereich, der das „Lebensrisiko Alter" reguliert. ${ }^{1}$ Was Lenhardt und Offe (1977) für diesen Bereich Mitte der 1970er Jahre noch als theoretisch mögliche Anpassungsstrategien des Sozialstaates beschrieben hatten, ist inzwischen weitgehend Realität: Das Rentenniveau und die Möglichkeiten des vorzeitigen Erwerbsausstiegs wurden reduziert, das Renteneintrittsalter erhöht. Diese Entwicklungen in beiden Regelungsmaterien (Zeitpunkt des Renteneintritts, Rentenhöhe) bedeuten Verschiebungen der Grenze zwischen Sozialpolitik und Tarifsystem. Hier kann aus Platzgründen nicht auf die einzelnen Etappen der jeweiligen Grenzverschiebungen eingegangen werden (vgl. dazu Fehmel 2012; Fröhler et al. 2013). Festzuhalten bleiben aber ihre strukturellen Effekte. Der Rückzug des Staates aus der Regelungsmaterie „flexibler Erwerbsausstieg“ zwingt die Sozialpartner zu verstärkten Bemühungen um tarifliche Regulierung. Vom politischen System werden sie darin ermutigt: Bei allen parteipolitischen Differenzen im Detail ist den aktuellen Positionen der Parteien gemein, dass die Tarifvertragsparteien als zukünftig maßgebliche Gestalter des Erwerbsausstiegs adressiert werden, dass also letztlich die Regelungskompetenz hinsichtlich des Rentenübergangs an die Tarifpartner delegiert wird. Auch die Abkehr der Politik vom Imperativ der lebensstandardsichernden gesetzlichen Rente hatte für die Verbände der Industriellen Beziehungen weitreichende Folgen. Jahrzehntelang waren sie an der politischen Gestaltung der Gesetzlichen Rentenversicherung (GRV) beteiligt, über die Selbstverwaltung der Rentenkassen ebenso wie über parteipolitische personale Verflechtungen und Einbindungen in Regierungstätigkeit. Durch den Paradigmenwechsel wurde die Bedeutung der Sozialpartner in der politischen Arena geschwächt, zugleich aber ihre Bedeutung in der tarifautonomen Arena gestärkt (Wiß 2011). Im Zuge der sozialpolitischen Durchsetzung des Drei-Säulen-Modells (gesetzliche Rente, betriebliche und private Altersvorsorge) wurde seit 2002 die Möglichkeit der betrieblichen und tariflichen Altersvorsorge als einer Säule des Altersvorsorge-Mixes - und damit die Rolle der Tarifpartner und der betrieblichen Akteure bei der Alterssicherung - deutlich aufgewertet. Beide Beispiele zeigen: Der Befund, dass das wohlfahrtsstaatlich institutionalisierte Alterssicherungssystem eine hohe Instrumentalisierbarkeit für tarifliche und betriebliche Zwecke aufweist (Rosenow 2000, S. 144), hat sich in sein Gegenteil verkehrt. Nunmehr werden das Tarifsystem und die betrieblichen Sozial- und Konflikträume für staatliche Zwecke des sozialpolitischen Rückzugs in Anspruch genommen.

\section{Die „Vertariflichung“ sozialer Sicherheit}

\subsection{Folgen für die Gewerkschaften}

Damit gehen zentrale sozialpolitische Verantwortlichkeiten, nämlich die für die Regulierung des Rentenübergangsgeschehens und für das Gesamtversorgungsniveau im Ruhestand, zu einem großen Teil vom Staat auf die Verbände über - einschließlich der damit verbundenen Legitimitätsri-

1 Die anderen großen, mittels Sozialversicherungen sozialpolitisch bearbeiteten Lebensrisiken (Arbeitslosigkeit, Krankheit, Arbeitsunfallfolgen/Behinderung und Pflegebedürftigkeit) sind in Deutschland bislang nicht oder kaum Gegenstand tariflicher Regulierung. Und die wenigen bestehenden Tarifregelungen zur Abfederung der materiellen Folgen der genannten Risiken sind nicht substitutiv zu sozialstaatlichen Leistungen, sondern komplementär auf oft nur niedrigem Niveau. 
siken. Durch seinen Rückzug zwingt der Staat den Sozialpartnern - und hier insbesondere den Gewerkschaften neue Themen und Tätigkeitsfelder auf, auf denen Letztere sich relativ frei bewegen können. Was sich daraus ergibt, ist staatlich gesteuerte Tarifautonomie (Fehmel 2010). Andererseits bietet sich den Gewerkschaften dadurch auch die Chance der organisationalen Festigung (Trampusch 2007, S. 264): Im Zuge ihres Bedeutungszuwachses für soziale Sicherung können sie für potenzielle Mitglieder attraktiver werden. Auch könnten sie ihre tarifsozialpolitischen Leistungen stärker als bislang an den Mitgliedschaftsstatus binden. In einigen Gewerkschaften - namentlich in solchen, die mit starken Mitgliedschaftsproblemen und mit starken Konkurrenzgewerkschaften zu kämpfen haben - wird das ganz offen debattiert. Anders als üblicherweise tarifliche Einkommenspolitik wird Tarifsozialpolitik als selektiver Anreiz, als exklusives, Mitgliedern vorbehaltenes Gut thematisiert.

Das setzt natürlich voraus, dass die Gewerkschaften ihren Mitgliedern auch attraktive Tarifsozialpolitik anbieten können. Damit sind die sehr unterschiedlichen Handlungsbedingungen der einzelnen Gewerkschaften in Deutschland angesprochen. Auf die diesbezüglichen Details kann hier ebenso wenig eingegangen werden (vgl. dazu Wiß 2012; Fehmel 2011) wie auf die dafür relevanten strukturellen Entwicklungen am Arbeitsmarkt und im System der Industriellen Beziehungen in den letzten Dekaden, die insgesamt geprägt sind von abnehmender gewerkschaftlicher Verhandlungs- und Kampffähigkeit, von um sich greifender Tarifflucht der Arbeitgeber oder auch vom massiven und politisch gewollten Ausbau eines tariflich kaum regulierten Niedriglohnsektors (Müller-Jentsch 2007). Als übergreifender Trend zeigt sich damit: Erstens sind die Chancen für Beschäftigte, von Tarifstandards - und damit auch von Tarifsozialpolitik - überhaupt erfasst zu werden, zunehmend ungleich verteilt. Das führt zu einer sich verschärfenden Selektivität des $\mathrm{Zu}$ gangs der Beschäftigten zu tariflich vermittelter sozialer Sicherheit (Bispinck 2012, S. 217). Und überall dort, wo eine tarifpolitische Gestaltung überhaupt möglich ist, differenzieren sich zweitens die Tarifstandards selbst erheblich aus. Mit der Entdifferenzierung zwischen Sozialpolitik und Tarifsozialpolitik geht also eine Differenzierung sozialer Sicherungsmöglichkeiten im Tarifsystem einher. Dies führt zu einer nachhaltigen Re-Strukturierung der Solidaritäts- und Loyalitäts-, also Umverteilungsbeziehungen innerhalb des Arbeitnehmerlagers. Denn die Re-Integration von Fragen sozialer Sicherung in das tarifliche und betriebliche Aushandlungsgeschehen erhöht nicht in erster Linie die Konfliktwahrscheinlichkeit zwischen „Arbeit“ und „Kapital“. Sie erhöht vielmehr die Wahrscheinlichkeit von Interessenkollisionen zwischen den Beschäftigten.

\subsection{Folgen für die Beschäftigten}

Um das zu begründen, ist ein kurzer Blick auf den Ablauf einer Tarifverhandlung und den Inhalt einer Tarifeinigung erforderlich. Der entscheidende Begriff hierbei lautet $\mathrm{Pa}$ - ketlösung. In der Öffentlichkeit wird darunter üblicherweise verstanden, dass sich eine Tarifeinigung zusammensetzt aus mehreren Komponenten, die im Idealfall aufeinander bezogen sind und die es beiden Verhandlungsseiten erlauben, Verhandlungserfolge zu kommunizieren. Vor diesem Hintergrund bedeutet die „Vertariflichung“ sozialer Sicherheit zunächst einmal, dass im Rahmen der Tarifautonomie bzw. der Sozialpartnerschaft zu den klassischen, unmittelbar arbeitsbezogenen Themen wie Einkommenshöhe, Einkommensstaffelung, Arbeitszeit, Beschäftigungsschutz etc. nun noch weitere Themen hinzukommen bzw. dass diese innerhalb des Themenpools deutlich an Bedeutung zulegen.

Intern meint der Begriff Paketlösung aber noch mehr. In der Bundesrepublik hat sich zwischen den großen Gewerkschaften und den Arbeitgeberverbänden ein Verfahren etabliert, das im Wesentlichen wie folgt abläuft: Zunächst wird in Tarifverhandlungen (mal mehr, aber meistens weniger konfliktreich) ein Gesamtverteilungsvolumen festgelegt, also ein Steigerungssatz der Gesamtarbeitskosten. Wie hoch diese Steigerung ist, ist abhängig von den jeweiligen Machtverhältnissen in einer Branche. Die Erfahrung der letzten Jahrzehnte zeigt jedoch, dass der Steigerungssatz sich üblicherweise in der Nähe der sogenannten Meinhold-Formel (Summe aus Produktivitäts- und Preissteigerungen) einpegelt. Wenn über die Steigerung des Gesamtvolumens zwischen Arbeitgeberseite und Gewerkschaft Einigkeit erzielt ist, wird in einem zweiten Schritt darüber verhandelt, welchen Anteil die einzelnen Paket-Komponenten am Gesamtpaket haben sollen. Im Zuge der „Vertariflichung“ sozialer Sicherheit nimmt nun zwar die Zahl möglicher Komponenten des Gesamttarifpakets zu, nicht aber dessen Gesamtvolumen. Vielmehr verschiebt sich die Gewichtung der Komponenten. Bei gedeckeltem Gesamtverteilungsvolumen zwingt die Aufnahme zusätzlicher Komponenten zur Beschneidung anderer Komponenten, z. B. der Entgeltsteigerung.

An der Entscheidung darüber, zu welchen Anteilen das vereinbarte Gesamtsteigerungsvolumen auf die einzelnen Komponenten verteilt wird, sind die Arbeitgeber zwar nicht unbeteiligt, aber im Wesentlichen obliegt sie den Gewerkschaften. Die geraten damit in eine Situation, die ich das Volumen-Dilemma nenne: Weil den Gewerkschaften für reale Ausweitungen des Verteilungsvolumens zur zusätzlichen Befriedigung sozialer Sicherungsbedürfnisse die Durchsetzungskraft fehlt (und den Arbeitgebern der Wille), muss nun ein relativ feststehendes Verteilungsvolumen für die Befriedigung der Lohnsteigerungswünsche und der Sicherungsbedürfnisse der Beschäftigten ausreichen. Problematisch ist dies deshalb, weil diese Bedürfnisse und Interessen unter den Beschäftigten ungleich verteilt sind. So stehen etwa Geringverdienern mit klaren Konsum- und damit Entgelt(erhöhungs)präferenzen Bezieher mittlerer und hoher Einkommen mit ambivalenten Einstellungen oder gar mehr oder weniger deutlichen Sparpräferenzen, beispielsweise im Rahmen betrieblicher Altersvorsorge, gegenüber. Ältere Beschäftigte erwarten tarifliche Lösungen, mit denen ihre Ar- 


\section{Erwartungen der Arbeitnehmer an die Tarif(sozial)politik}

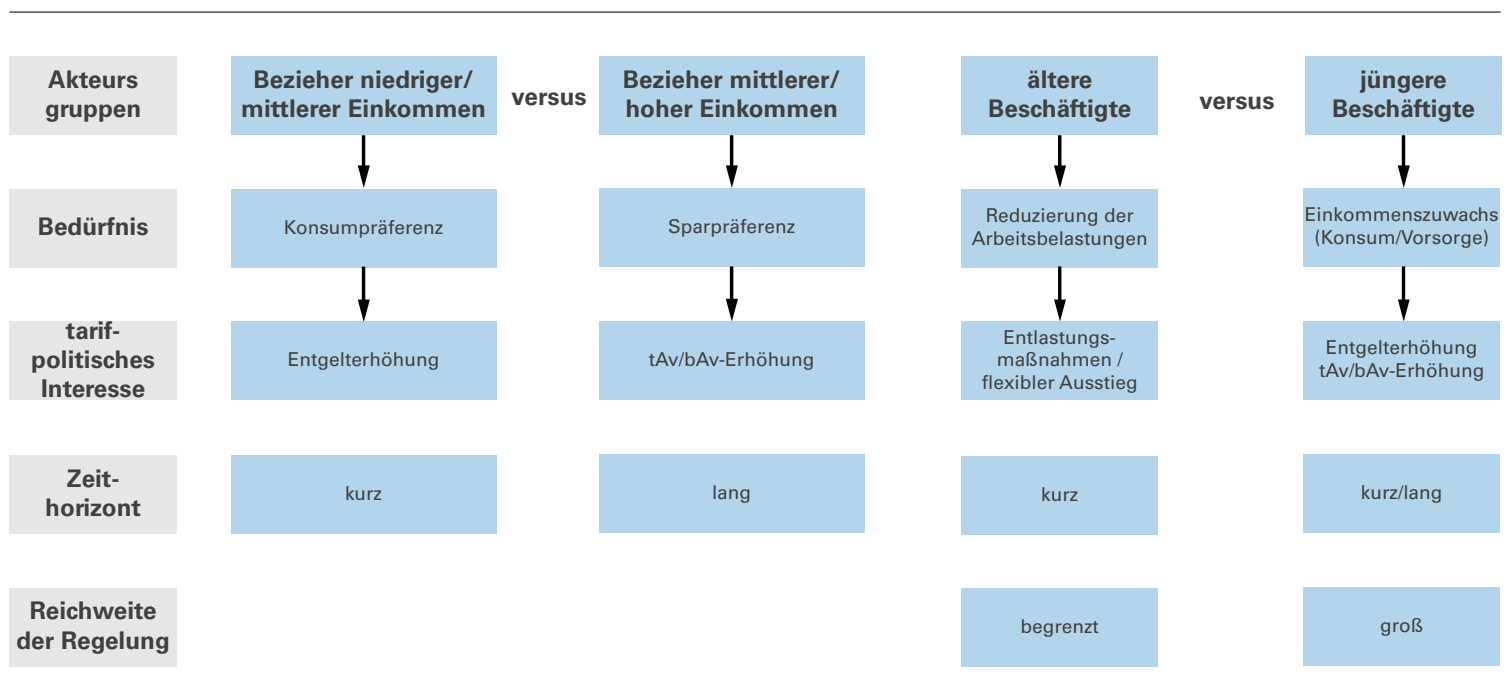

Quelle: Darstellung des Autors.

beitsbelastungen reduziert und/oder vorzeitige bzw. flexible Rentenübergänge ermöglicht werden; jüngere Beschäftigte stehen diesen in der Regel sehr teuren Maßnahmen oft skeptisch gegenüber und halten die Verwendung der Verteilungsmasse für Entgeltsteigerungen oder langfristig angelegte Vorsorgepläne für sinnvoller (Abbildung 1). Darüber hinaus führt die Aufwertung der betrieblichen Altersvorsorge (v. a. seit dem Jahr 2002 durch das Altersvermögensgesetz) auch zur Herausbildung unterschiedlicher Nutzergruppen mit jeweils verschiedenen Zielsetzungen und Möglichkeiten (Burger 2012). Durch die Integration sozialpolitischer Themen in die Tarifpolitik steigt also insgesamt die (innerhalb des Tarifsystems zu bearbeitende) Interessenheterogenität bei den Beschäftigten - und damit auch die Wahrscheinlichkeit von Interessenkollisionen.

\subsection{Folgen für die Solidaritätsbereitschaft}

Welche Folgen hat die beschriebene Entwicklung für das Solidarverhalten der Beschäftigten? Es gibt eine Vielzahl unterschiedlichster alltagssprachlicher Interpretationen und sozialwissenschaftlicher Definitionen des Begriffes Solidarität (Prisching 2003). Aber allen gängigen Auffassungen zufolge setzt solidarisches Handeln, erstens, ein gewisses $\mathrm{Maß}$ an Gleichartigkeit und an gleichartigen Interessen und Bedürfnissen voraus; zweitens, ein darauf aufbauendes gewisses Maß an Zusammengehörigkeit und Verbundenheit und gemeinsamer Identität, also Gruppenidentität, sowie, drittens, ein gewisses Maß an Umverteilungsbereitschaft innerhalb dieser Gruppe. Für die Erklärung der Motive solidarischen Verhaltens bediene ich mich eines pragmatischen Solidaritätsverständnisses. Zweifellos gibt es so etwas wie eine moralische Verpflichtung zur Hilfe. Handlungstheoretisch gehaltvoller dürfte aber ein Verständnis sein, das Solidarverhalten als eine spezifische Form der individuellen Interessenverfolgung betrachtet (Vobruba 1983, S. 72ff.): Man zeigt sich als Mitglied einer Gruppe solidarisch, d. h. zu eigenen Lasten umverteilungsbereit, weil man absehen oder jedenfalls nicht ausschließen kann, zu einem späteren Zeitpunkt auf die Bereitschaft anderer Gruppenmitglieder zur Umverteilung zu eigenen Gunsten angewiesen zu sein. In diesem Sinne ist solidarisches Handeln eigennützig, und es bedeutet keineswegs den Verzicht auf einen individuellen Interessenstandpunkt. Es basiert auf grundlegenden Reziprozitätsnormen und es impliziert einen relativ weiten Zeithorizont des Handelns aller Gruppenmitglieder.

Dennoch besteht grundsätzlich immer auch die Möglichkeit des Ausscherens, d. h. die Möglichkeit kurzfristiger, asolidarischer, individueller Interessenverfolgung. In solchem Verhalten steckt das Potenzial für Entsolidarisierungsdynamiken. Wenn solidarisches Verhalten zwingend ein in Gruppen gezeigtes und auf Gruppen bezogenes Verhalten ist, dann kann sich Entsolidarisierung generell in zweierlei Form äußern: Sie führt entweder zu faktischer Dekollektivierung, also zur Auflösung der Gruppe als Reziprozitätsund Solidarverband und zum Umschalten auf (kurzfristige) individuelle Verfolgung von Eigeninteressen. Oder Entsolidarisierungsdynamiken forcieren die Re-Definition gemeinsamer, solidarisch zu verfolgender Interessen und ggf. gar die Re-Definition von Gruppenzugehörigkeit. Exakt das sind die beiden größten organisationalen Herausforderungen, mit denen sich die großen Gewerkschaften gegenwärtig konfrontiert sehen. Sie haben es einerseits zu tun mit zu- 
nehmender individueller Interessenverfolgung, erkennbar an der steigenden Zahl von Beschäftigten, die sich gegen eine Gewerkschaftsmitgliedschaft und damit implizit gegen eine kollektive, tarifliche Gestaltung von Arbeits-, Einkommens- und Absicherungsbedingungen aussprechen. Und die großen Gewerkschaften sehen sich andererseits bedenklichen Prozessen der Subgruppenbildung und Re-Definition von Gruppenzugehörigkeit gegenüber, erkennbar vor allem in der Verbreitung diverser, sehr durchsetzungsfähiger Kleinst- und Spartengewerkschaften mit deutlich homogeneren Interessen ihrer Mitglieder (Schroeder et al. 2011).

Die Vertariflichung sozialer Sicherung erhöht also insgesamt die Relevanz von Interessenunterschieden. Dies und die Bedeutungszunahme unterschiedlicher Zeithorizonte bei den Beschäftigten bzw. den Mitgliedern begünstigt Entsolidarisierungsprozesse innerhalb des Kreises der Arbeitsmarkt-Insider.

\section{Verteilungspolitische Konsequenzen}

Im Rahmen staatlicher Sozialpolitik gab und gibt es solche Interessendifferenzen der versicherten Arbeitnehmer natürlich auch. Aber der Staat hat dieses Problem durch die Einführung der Sozialversicherungspflicht gelöst, also letztlich durch eine Form der Zwangssolidarität. Über diese Möglichkeit verfügen die Gewerkschaften nicht. Sie müssen diskursiv und dialogisch die zunehmend heterogenen Interessen verschiedener Beschäftigtengruppen integrieren und Interessenkollisionen zu Interessenkompromissen verarbeiten (Hyman 2011). Für durchsetzungsstarke Gewerkschaften in Hochlohn-Branchen bestehen hier sicher zahlreiche Möglichkeiten, etwa durch Einführung von Arbeitgeber-Obligatorien oder mit Optionsmodellen im Rahmen von Demografie-Tarifverträgen. Es gibt jedoch begründete Zweifel, dass Vergleichbares allen Gewerkschaften problemlos gelingt. Auf Basis empirischer Befunde ${ }^{2}$ lässt sich etwas sagen zu den Wahrscheinlichkeiten, wie diese Interessenkonflikte wohl überwiegend gelöst werden: Aufgrund der unterschiedlichen Reichweite quantitativer und qualitativer Tarifergebnisse (von Entgelterhöhungen profitieren „alle“ und sofort, von tariflichen Sozialleistungen vermeintlich nur Subgruppen und später) und der betroffenheitsorientierten Mobilisierungsbereitschaft der Gewerkschaftsmitglieder gerät in Tarifrunden und Arbeitskämpfen qualitative Tarifpolitik regelmäßig ins Hintertreffen.

Das hat verteilungspolitische Konsequenzen: Wenn sich der Gesetzgeber aus der unmittelbar redistributiv wirkenden Gestaltung sozialer Sicherung auf die Regulierung nichtstaatlicher Wohlfahrtsproduktion zurückzieht, die Tarifparteien aber aus den beschriebenen Gründen zu einer vollständigen Kompensation dieser sozialpolitischen Entstaatlichung weder in der Lage noch willens sind, dann kommt zwangsläufig den einzelbetrieblichen und den in- dividuellen Bedingungen, Ressourcen und Restriktionen immer mehr Gewicht zu (Berner 2009). D. h. für die Befriedigung individueller Sicherungsbedarfe gewinnen die einzelbetrieblichen Aushandlungsbedingungen und Gestaltungsmöglichkeiten ebenso an Bedeutung wie die jeweiligen individuellen Merkmale der Arbeitnehmer, also die Erwerbsund Versicherungsbiografie, der Erwerbsstatus, das (Haushalts-)Einkommen, Geschlecht, die Gesundheit, Qualifikation u. ä. (Blank 2012, S. 187f.). Im Ergebnis führt die „Vertariflichung“ von Sozialpolitik zu zunehmend ungleich verteilten Chancen der Beschäftigten auf den Zugang zu sozialer Absicherung und zu zunehmend ungleichen nichtstaatlichen Sozialleistungen selbst - und damit letztlich zu einem Anstieg der sozialen Ungleichheit.

Damit ist die „Vertariflichung“ der Wohlfahrtsproduktion anschlussfähig an die eingangs genannten beiden Trends des Sozialstaatswandels in Deutschland. Sie fungiert zudem aufgrund ihrer beschriebenen verteilungspolitischen Konsequenzen gleichsam als Trendbeschleuniger. Die Gewerkschaften sind gezwungen, den Interessenschwerpunkt der unteren Einkommensgruppen an maximaler Einkommenserhöhung zur Verbesserung der gegenwärtigen Konsummöglichkeiten zu beachten und in die Dominanz quantitativer Tarifpolitik zu übersetzen. Wollen sie dabei ihren integrativen und nivellierenden Anspruch nicht aufgeben, so erschwert das eine gesonderte qualitative Tarifpolitik für die Beschäftigten oberer Einkommensgruppen. Damit wird qualitative Tarifpolitik zwangsläufig nachrangig. Für die Gutverdiener ist dies weitgehend unproblematisch oder gar vorteilhaft: Sie sind in der Lage, ihren individuellen Präferenzen folgend und unabhängig von kollektivvertraglichen Vorgaben den sparfähigen Teil ihres hohen Einkommens in individuelle Sicherungs- und Altersvorsorgestrategien umzuleiten. Das begünstigt die „Vermarktlichung“ sozialer Sicherung. Bezieher niedriger Einkommen, bei denen die Erfolge quantitativer Tarifpolitik überwiegend in den Konsum fließen, haben hingegen deutlich weniger Möglichkeiten einer individuellen marktförmigen Risikoabsicherung. Das treibt vor dem Hintergrund des stattfindenden Umbaus sozialstaatlicher Instrumente langfristig die Dualisierung sozialer Sicherung und die Ausbreitung staatlicher Mindestsicherungsformen voran. Damit aber wird die etablierte Insider/Outsider-Unterscheidung brüchig: Es ist absehbar, dass unter den gegebenen Bedingungen des politisch gewollten Bedeutungszuwachses von Tarifsozialpolitik nun vermehrt auch ein Normalarbeitsverhältnis keine Garantie mehr ist gegen die individuelle Abhängigkeit von Grundsicherungsleistungen jenseits des Sozialversicherungsprinzips.

2 Vgl. z. B. die Ergebnisse des von der Hans-Böckler-Stiftung geförderten Forschungsprojektes "Re-Flexibilisierung des Rentenübergangs" am Institut für Soziale Arbeit und Sozialpolitik der Universität Duisburg-Essen (Fröhler et al. 2013). 


\section{LITERATUR}

Behrens, M. (2005): Mitgliederrekrutierung und institutionelle Grundlagen der Gewerkschaften. Deutschland im internationalen Vergleich, in: Berliner Debatte Initial 16 (5), S. 30-37

Berner, F. (2009): Der hybride Sozialstaat. Die Neuordnung von öffentlich und privat in der sozialen Sicherung, Frankfurt a. M.

Bispinck, R. (2012): Sozial- und arbeitsmarktpolitische Regulierung durch Tarif vertrag, in: Bispinck, R./Bosch, G./Hofemann, K./Naegele, G. (Hrsg.): Sozialpolitik und Sozialstaat, Wiesbaden, S. 201-219

Blank, F. (2012): Die neue betriebliche Altersversorgung und ihre Nutzer, in: WSI-Mitteilungen 65 (3), S. 179-188, http://www.boeckler.de/wsimit_2012_03_ blank.pdf

Briefs, G. (1930): Der wirtschaftliche Wert der Sozialpolitik, in: Die Reform des Schlichtungswesens. Der wirtschaftliche Wert der Sozialpolitik, Schriften der Gesellschaft für Soziale Reform (83), Jena, S. 144-170

Burger, C. (2012): Die drei Formen betrieblicher Altersvorsorge in Deutschland, in: WSI-Mitteilungen 65 (3), S. 235-242, http://www.boeckler.de/wsi-

mit_2012_03_burger.pdf

Fehmel, T. (2010): Staatlich gesteuerte Tarifautonomie. Institutioneller Wandel durch semantische Kontinuität, in: Berliner Journal für Soziologie 20 (4), S. 423-445

Fehmel, T. (2011): Regelungen derTarif- und Betriebspartner zu flexiblen Altersübergängen, in: Soziale Sicherheit. Zeitschrift für Arbeit und Soziales 60 (2), S. 45-51

Fehmel, T. (2012): Weder Staat noch Markt. Soziale Sicherheit und die Re-Funktionalisierung des Arbeitsvertrages, in: Bach, M. (Hrsg.): Der entmachtete Leviathan. Löst sich der Staat auf?, in: Zeitschrift für Politik, Sonderband 12, S. 155-174

Fröhler, N./Fehmel, T./Klammer, U. (2013): Flexibel in die Rente. Gesetzliche, tarifliche und betriebliche Perspektiven, Berlin

Hall, P. A. (1993): Policy Paradigms, Social Learning, and the State. The Case of Economic Policymaking in Britain, in: Comparative Politics 25 (3), S. 275-296 Hockerts, H. G. (1998): Drei Wege deutscher Sozialstaatlichkeit. NS-Diktatur, Bundesrepublik und DDR im Vergleich, München, S. 7-25

Hyman, R. (2011): Gewerkschaftliche Strategien und Solidaritätspolitik unter globalen Konkurrenzbedingungen, in: Gerlach, F./Greven, T./Mückenberger, U. (Hrsg.): Solidarität über Grenzen. Gewerkschaften vor neuer Standortkonkurrenz, Berlin, S. 51-73

Leisering, L./Marschallek, C. (2010): Zwischen Wohlfahrtsstaat und Wohlfahrtsmarkt. Alterssicherung und soziale Ungleichheit, in: Hockerts, H. G. (Hrsg.): Soziale Ungleichheit im Sozialstaat. Die Bundesrepublik Deutschland und Großbritannien im Vergleich, München, S. 89-115

Lenhardt, G./Offe, C. (1977): Staatstheorie und Sozialpolitik. Politisch-soziologische Erklärungsansätze für Funktionen und Innovationsprozesse der Sozialpolitik, in: Ferber, C. v./Kaufmann, F.-X. (Hrsg.): Soziologie und Sozialpolitik, Opladen, S. 98-127

Lepsius, M. R. (1995): Soziale Symmetrie:Tarifautonomie und staatliche Sozialpolitik, in: Fricke, W. (Hrsg.): Jahrbuch Arbeit und Technik 1995: Zukunft des Sozialstaats, Bonn, S. 3-7
Müller-Jentsch, W. (2007): Strukturwandel der industriellen Beziehungen. „Industrial citizenship" zwischen Markt und Regulierung, Wiesbaden

Nullmeier, F. (2001): Sozialpolitik als marktregulative Politik, in: Zeitschrift für Sozialreform 47 (6), S. 645-668

Nullmeier, F. (2004): Vermarktlichung des Sozialstaats, in: WSI-Mitteilungen 57 (9), S. 495-500, http://www.boeckler.de/wsimit_2004_09_nullmeier.pdf Palier, B./Martin, C. (2007): From 'a Frozen Landscape' to Structural Reforms: The Sequential Transformation of Bismarckian Welfare Systems, in: Social Policy \& Administration 41 (6), S. 535-554

Prisching, M. (2003): Solidarität: Der vielschichtige Kitt gesellschaftlichen Zusammenlebens, in: Lessenich, St. (Hrsg.): Wohlfahrtsstaatliche Grundbegriffe. Historische und aktuelle Diskurse, Frankfurt a. M., S. 157-190

Rosenow, J. (2000): Karriere- und Lebenslaufpolitik in deutschen Unternehmen. Gesellschaftliche Rahmenbedingungen und politische Regulierungen, in: George, R./Struck, O. (Hrsg.): Generationenaustausch im Unternehmen, München, S. 141-157

Schroeder, W./Kalass, V./Greef, S. (2011): Berufsgewerkschaften in der Offensive. Vom Wandel des deutschen Gewerkschaftsmodells, Wiesbaden

Trampusch, C. (2007): Industrial Relations as a Source of Social Policy. A Typology of the Institutional Conditions for Industrial Agreements on Social Benefits, in: Social Policy \& Administration 41 (3), S. 251-270

Vobruba, G. (1983): Politik mit dem Wohlfahrtsstaat, Frankfurt a. M.

Vobruba, G. (1991): Jenseits der sozialen Fragen. Modernisierung und Transformation von Gesellschaftssystemen, Frankfurt a. M.

Vogel, B. (2007): Die Staatsbedürftigkeit der Gesellschaft, Hamburg

Wiesenthal, H./Clasen, R. (2003): Gewerkschaften in Politik und Gesellschaft. Von der Gestaltungsmacht zum Traditionswächter?, in: Schroeder, W./Weßels, B. (Hrsg.): Gewerkschaften in Politik und Gesellschaft der Bundesrepublik Deutschland, Wiesbaden, S. 296-322

Wiß,T. (2011): Der Wandel der Alterssicherung in Deutschland. Die Rolle der Sozialpartner, Wiesbaden

Wiß, T. (2012): Der Ausbau der betrieblichen Altersvorsorge: Soziale Ungleichheiten für Beschäftigte bei gleichzeitiger Wiederbelebung der Sozialpartner, in: Sozialer Fortschritt 61 (7), S. 165-172

\section{AUTOR}

THILO FEHMEL, Dr., ist Wissenschaftlicher Mitarbeiter am Lehrstuhl für Sozialpolitik des Instituts für Soziologie der Universität Leipzig. Arbeitsschwerpunkte: Soziologie der Industriellen Beziehungen, Soziologie der sozialen Sicherheit und Sozialpolitik.

fehmel@sozio.uni-leipzig.de 